\title{
Se traduire
}

\author{
Marguerite Andersen
}

\section{Translating Myself}

The writer speaks with a forked tongue: German is her birth language, French her mother tongue and English is the language of the society in which she lives. Each of these has a particular resonance: German is associated with fascism and masculinity; French is the inner language of the emotions, especially of motherhood; English is the language of friendship, the language into which she has translated her own text, Autrement pareille, so that her friends may read her work.

For a trilingual person, translation should be an easy task. It is not, however, since it obliges the writer / translator to enter twice into the fortifications of sexism in language(s), to encounter twice the emotions of creation. The first act of translation is that of the interior voice into writing. In the second, that of translating from French to English, while there has been a loss of the possibilities for foregrounding the feminine, there is also the discarding of the absurd gender-marked grammatical rules of French. And there are ways of marking the feminine in English after all. In translating one's own text, the translator relives the emotions of the text, in this case the painful emotions of the motherdaughter relationship. This second act of translation is as complex as the first whose process it repeats.

Quand on a, comme moi, la langue fourchue, coupéeen trois, une partie allemande, langue natale, une partie française, langue maternelle, et une partie anglaise, langue parlée autour de moi et par moi, traduire les autres et surtout se traduire soi-même devrait au fond être assez facile. Et pourtant il n'en est rien. Je viens d'en faire l'expérience, ayant, sur le conseil de Barbara Godard, traduit en anglais mon recueil de poèmes en prose, L'Autrement pareille.

Quelle est ma position vis-à-vis de mes trois langues, quels sont mes sentiments? Je me vois incapable de débarrasser mon anglais parlé d'un accent allemand qui me gêne mais contre lequel je n'ai 


\section{Se traduire $\cdot 73$}

jamais rien fait, peut-être pour ne pas renier totalement une origine qui, dans unsens, megêne, elle aussi. Ilest rarequeje parleallemand, il est plus rare encore que je l'écrive. Je fais partie d'une génération qui n'a pas collaboré avec Hitler mais qui ne peut se défaire de lui. Dans mon cas, cela se traduit par l'abandon presque total de la langue de mon enfance qui, pour moi, ne peut perdre ses connotations fascistes, militaristes, et essentiellement masculines. Quant à l'anglais, c'est pas une langue que j' aime bien, que je parle bien, accent ou pas, quej'écris bien aussi. Mais je préfère le français que j'appelle ma langue maternelle parce que ma mère a pris soin que je l'apprenne, parce que j'ai élevé trois enfants dans cette langue, que c'est la langue dans laquelle je m'entretiens avec mes six petites-filles et aussi avec le petit-fils, venu dernièrement mettre en évidence toute cette descendance féminine. Je suis donc tendrement liée au français. Le français est ma langue intérieure, celle qui résonne avec le plus de volupté dans mon corps. C'est elle que j'écoute quand j'écris, c'est d'elle que je me sers pour faire de ma voix intérieure un texte écrit.

Cette première traduction de la voix intérieure vers le texte, vers la langue extérieure, est particulièrement difficile quand on est femme. En se disant, la femme se découvre étrangère dans un monde mâle, et si elle veut se faire entendre, dans ce monde qui au fond exige $d^{\prime}$ elle surtout le silence, elle n'a pour le faire qu'une langue mal / mâle construite. Se servir de cette langue pourrait signifier se faire mal / mâle, exige une vigilance tout à fait particulière, demande qu' on se fasse critique, voleuse et innovatrice en même temps. Sans le féminisme, sans le cercle des femmes / amies, sans la spirale de nos désirs et de nos textes où nos paroles se rencontrent, aurais-je pu écrire? Non. Sans ma mère et sa volonté de me faire apprendre le français dans lequel elle voyait pour moi une porte desortie, aurais-je pu écrire? Non encore.Je dois mes textes à ma mère et à mes compagnes. Grâce à elles, j'ai pu traduire ma voix intérieure. Et malgré le sexisme ordinaire du français, j'ai pu grâce à elles y découvrir des mots à aimer, faire de la mère maternelle une mer bleue, faire chanter les voyelles, jouir du féminin des mots: fille, pareille, ré-elle, joie, tendresse, larme, montagne, neige, lèvres, langue, peau, eau, île, utopie ... J'ai appris à éviter le masculin, à chercher le mot juste, au féminin, dire pelouse plutôt que gazon, route plutôt que chemin. J'ai appris à faire valoir le féminin malgré tout, mais sans perdre de vue le fait que s'écrire, c'est $\mathrm{s}^{\prime}$ exprimer dans la langue del' autre, dans la langue del' homme. Donc sans perdre la colère ou l'élan féministes. 
Traduis donc ton texte toi-même, me disait Barbara, et j'en avais envie, puisque des amies anglophones regrettaient de ne pas pouvoir me lire et que je voulais leur faire plaisir. En même temps, j'en avais peur, sans bien savoir pourquoi. Finalement je me suis dit que, oui, ce serait un jeu d'enfant, un simple exercice d'acrobatie intellectuelle et linguistique, que je n'avais rien à craindre, puisque la première traduction, celle de la voix intérieure vers l'écrit, était déjà faite.

Erreur. Se traduire dans une autre langue, faire en tant que femme la traduction de son propre texte, $c^{\prime}$ est entrer une deuxième fois dans les fortifications du sexisme des langues. C'est encore une fois se rendre compte de la solidité des obstacles et, bien que cela finisse par raviver le féminisme de la traductrice, c'est fondamentalement douloureux, irritant, pesant. Traduire son propre texte, $c^{\prime}$ est se heurter doublement.

D'un autre côté, le maniement de différentes langues permet de saisir leurs secrets favorables à la femme. En français, il est possible de faire ressortir le féminin, de le faire résonner, amie, pareille, merveille ... Traduisez cela en anglais et le féminin s'évanouit. Toutefois, la grammaire anglaise $n^{\prime}$ impose aucune des ces absurdités que celle du français prescrit. Trois cents femmes et un moustique, qui en français doivent devenir 'ils,' sont en anglais tout simplement 'they.' Il n'y a pas en anglais cet accord qui efface le féminin sur lequel le masculin l'emporte, comme par exemple dans la phrase: 'Anne et Pierre, amants heureux, se sont rencontrés au clair de lune ...' En anglais, a writer is a writer, she or he, her mother is the mother of a female child, alors que les mots 'sa mère' ne nous disent rien sur le sexe de l'enfant, mot masculin d'ailleurs ce qui entraîne bien sûr toutes sortes $d^{\prime}$ accords où le masculin encore une fois l'emporte sur le féminin.

Traduire son propre texte d'une langue à l'autre, c'est faire des découvertes heureuses: cherchant à traduire l'expression française 'mère du vinaigre,' $j$ ' en fais un 'mother-of-pearl,' meublant ainsi au féminin mon univers trilingue. Je $m$ 'aperçois que des mots figés en français dans le genre masculin - cordon ombilical, ventre, rêve-peuvent en anglais, dans mon imagination, revêtir bien plus facilement des connotations féminines. Et mêmeuneonomatopée comme 'hush,' dont je me sers pour traduire le 'non' par lequel débute mon texte en français (et que j'avais préféré au 'chut' qui m'avait semblé dur et abrupt dans sa tonalité), résonne dans mon oreille avec une prolongation douce et essentiellement féminine.

Se traduire soi-même, c'est aussi vivre et traduire une même 
émotion deux fois. Avec L'Autrement pareille, qui traite de la relation mère-fille, je croyais avoir surmonté par l'écriture ce qui m'avait tourmentée dans cette relation, je pensais donc pouvoir faire la traduction anglaise de ce texte tout simplement, intellectuellement, froidement et sans verser de larmes. Erreur encore.

Est-ce parce que la relation mère-fille est tellement forte? Parceque l'émotion ne saurait se traduire sans émotion? Parce que pour une féministe émotion et pensée font partie du même rêve essentiel, comme nous le dit Nicole Brossard dans Picture Theory? Ce sont là des questions que je me pose aujourd' hui, après avoir terminé le travail ... dont voici un échantillon:

Journée-désastre, pleurs intarissables devant le départ de la bien-aimée qui me quitte sans savoir comment. Mère de sel, mère de vinaigre, je voudrais pleurer une mer / mère de larmes de joie, ouvrir grande la porte comme je me suis ouverte il y a vingt ans, mais je serre les muscles, $l^{\prime}$ adultement ne se fait pas de façon aussi naturelle que l'enfantement, faut-il donc cette fois-ci vraiment employer les forceps et pourquoi, la déchirure necesse de s'échancrer, le cordon ombilical se noue autour de ma gorge, aucun cri ne se forme dans ma bouche, ma respiration devient imperceptible, peut-être, si je ne bouge pas, aurai-je la force de devenir mille fois plus forte, peut-être l'immobilité fera-t-elle geler mes larmes, peut-être saurai-je devenir finalement femme frigide, m'insensibiliser, me désensibiliser, mourir (23).

Time of disaster, endless tears upon the departure of the beloved who is leaving without knowing how. Mother of salt, mother of pearl, I want to cry the mother sea of joyful tears, open wide the doors as I opened myself twenty years ago, but I tense my muscles, adult-birth does not happen as readily as childbirth, must the forceps be used this time and why, the tear does not stop tearing, the umbilical cord is strangling me, in my throat no cry can be formed, my breathing becomes imperceptible, perhaps, if I stop moving will I have the strength to becomes a thousand times stronger, perhaps immobility will freeze my tears, perhaps I will finally become the frigid woman, insensitive, desensitized, dead.

\section{Works Cited}

Andersen, Marguerite. Autrement pareille. Sudbury: Prise de Parole, 1984. 